\title{
Anaemia and the risk of progression from non-proliferative diabetic retinopathy to vision threatening diabetic retinopathy
}

\author{
Yafeng $\mathrm{Li}^{1} \cdot$ Yinxi Y $\mathbf{u}^{2} \cdot$ Brian L. VanderBeek $k^{1,3,4}$
}

Received: 10 April 2019 / Revised: 13 July 2019 / Accepted: 28 July 2019 / Published online: 4 October 2019

(c) The Author(s), under exclusive licence to The Royal College of Ophthalmologists 2019

\begin{abstract}
Background/Aims To determine if anaemia and oxygen delivery-related co-morbidities (ODCs) affect progression from non-proliferative diabetic retinopathy (NPDR) to vision-threatening diabetic retinopathy (VTDR).

Methods This is a retrospective cohort study using medical claims data from a large US insurer. Cohorts were created from all NPDR patients between 2002 and 2016. Primary exclusion criteria consisted of any previous diagnosis of proliferative diabetic retinopathy (PDR), diabetic macular oedema (DME) or treatment used in the care of VTDR. The main outcome was a new diagnosis of VTDR (DME or PDR), PDR, or DME. A time-dependent, multivariate Cox proportional hazard regression was used to determine the association between anaemia and other ODCs with NPDR progression.

Results Of the total 69,982 NPDR patients included for analysis, 12,270, 2,162, and 10,322 progressed to VTDR, PDR and DME, respectively. Both mild and moderate/severe (mod/sev) anaemia were associated with an increased hazard for progression to VTDR (mild HR:1.10, 95\% CI:1.04-1.16, $p<0.001$; mod/sev HR:1.20, 95\% CI:1.12-1.29, $p<0.001$ ), PDR (mild HR:1.29, 95\% CI:1.13-1.46, $p<0.001$; mod/sev HR:1.43, 95\% CI:1.21-1.69, $p<0.001$ ), and DME (mild HR:1.06, 95\% CI:1.00-1.13, $p<0.001$; mod/sev HR:1.14, 95\% CI:1.05-1.24, $p<0.001)$. ODCs such as chronic pulmonary disease and history of blood disorder/cancer were also significantly associated with an increased hazard for NPDR progression (HR $>1.00, p<0.001$ for all comparisons).

Conclusions Anaemia, independent of kidney disease, appears to play a significant role in progression from NPDR to VTDR, PDR, or DME. Concurrently, association of ODCs with NPDR progression lends support to the underlying mechanisms of anaemia in the pathogenesis of diabetic retinopathy.
\end{abstract}

Supplementary information The online version of this article (https:// doi.org/10.1038/s41433-019-0617-6) contains supplementary material, which is available to authorized users.

Brian L. VanderBeek

Brian.VanderBeek@uphs.upenn.edu

1 Scheie Eye Institute, Department of Ophthalmology, Perelman School of Medicine at the University of Pennsylvania, Philadelphia, PA, USA

2 Center for Preventive Ophthalmology and Biostatistics, Perelman School of Medicine at the University of Pennsylvania, Philadelphia, PA, USA

3 Center for Clinical Epidemiology and Biostatistics, Department of Biostatistics \& Epidemiology, Perelman School of Medicine at the University of Pennsylvania, Philadelphia, PA, USA

4 Leonard Davis Institute, Perelman School of Medicine at the University of Pennsylvania, Philadelphia, PA, USA

\section{Introduction}

Anaemia is a less frequently recognized co-morbidity of diabetes that may have an adverse effect on progression of diabetes-related microvascular complications [1]. Impaired renal erythropoietin release, reduced red cell survival, systemic inflammation and autonomic neuropathy all work in concert to make anaemia highly prevalent among patients with diabetes [1]. What is less understood is how this reduced capacity to deliver oxygen exacerbates the disease process with diabetics, especially for tissues with concurrent microvascular insults. Specific to progression of diabetic retinopathy, anaemia was initially evaluated over 30 years ago [2], but surprisingly little has been done since to comprehensively study the issue.

In 1985, the Diabetic Retinopathy Study found haemoglobin to be the second most predictive systemic factor in progression to severe vision loss in patients with diabetic retinopathy [2]. Later in 1997, a cross sectional, population- 
based study showed an association between haemoglobin levels below $12 \mathrm{~g} / \mathrm{dl}$ and progression to high risk proliferative disease [3]. This finding was confirmed by the ETDRS group a year later [4], which found that low haematocrit values at baseline were associated with a 5-year progression to proliferative disease. More recently, two smaller cross-sectional studies found associations between haemoglobin and diabetic retinopathy severity [5, 6], including the first to assess diabetic macular oedema (DME); but given the cross-sectional nature of the studies, a temporal relationship could not be inferred.

Oxygen delivery is essential to counter-acting diabetic microvascular ischemia. In this study, we postulate that systemic diseases in which the ability to deliver oxygen to the retina (termed here oxygen-delivery-related-comorbidities, or ODCs) is reduced can impact the hazard of NPDR patients progressing to vision-threatening diabetic retinopathy (VTDR). These states include not only anaemia, but other diseases that can affect oxygenation of blood like chronic pulmonary disease and blood disorder/cancer. Furthermore, anaemia itself is not a static variable. Assessing this variable 5 years prior to evaluating an outcome may lose important interval changes in the haemoglobin levels, masking the true association between anaemia and VTDR. For these reasons, we aim to perform a timeupdating cohort analysis of NPDR patients and assess the impact of ODCs on the risk for progression to VTDR and its component disease states, PDR and DME.

\section{Methods}

\section{Dataset}

The de-identified Clinformatics ${ }^{\mathrm{TM}}$ Data Mart Database (OptumInsight, Eden Prairie, MN) was used for this study. This database contains all of the outpatient medical claims (office visits, procedures, and medications given) as well as demographic data and some laboratory values for all patients enrolled in a large managed care network from across the USA. The subset of data available for this study included all patient visits from January 1, 2002 to December 31, 2016 within the database. Due to the de-identified nature of the database, the University of Pennsylvania's Institutional Review Board deemed this study exempt from review.

\section{Cohorts}

This is a time-updating cohort study evaluating the progression of NPDR. All patients who were diagnosed with NPDR based on ICD9 and ICD10 coding and had a history of diabetes mellitus were included within this study.
Previous studies of diabetic retinopathy diagnosis and treatment codes have been demonstrated to be valid for research purposes [7-9]. Similarly, patients were also mandated to be over 18 years old and have at least 24 consecutive months in the plan. Due to the time-updating nature of this study, the index date for each patient was considered the first date that a patient had both a diagnosis of NPDR and a recorded laboratory value of interest within their time in the plan.

Individuals were excluded if, at any time prior to the index date, they had a diagnosis of a more severe form of diabetic retinopathy, including PDR or DME. Other diseases that were within 120 days of the index date had their index date shifted to the first date of NPDR diagnosis after the 120-day window (See Supplemental Table 1 for all diagnosis, procedure, and drug codes used in the study).

\section{Covariates}

Numerous risk factors were assessed for their potential impact on the association of disease progression. All variables that were able to be assessed in a time-varying manner were updated every time new information was available. The primary covariates to be assessed were thought to be associated with oxygen delivery, anaemia and chronic pulmonary disease. Anaemia was based on haemoglobin level and stratified according to the World health Organization's level of anaemia (Males: normal $\geq 13.0$, mild $=$ 11.0-12.9, moderate/severe $\leq 10.9$; Women: normal $\geq 12.0$, mild $=11.0-11.9, \quad$ moderate/severe $\leq 10.9) \quad[10] . \quad$ The demographic variables that were not time-varying were assessed at the index date and include race, gender, region of the country, yearly income, and education level. Other updating variables assessed include age, calendar year and clinical variables: hypertension, hypercholesterolemia, ischemic stroke or transient ischemic attack, intracerebral haemorrhage, chronic liver disease, peripheral vascular disease, malignancy, and blood disorders. The Diabetic Complications Severity Index (DCSI) was used and is a diagnostic code-based severity index shown to better control for severity of the underlying diabetic disease state [11]. This score is created from six categories of diabetic complications using outpatient ICD9/ICD10 codes and applied to each patient. This metric has been validated and found to predict clinically relevant outcomes more accurately than traditional markers of diabetic severity such as glycosylated haemoglobin and duration of disease [11]. By DCSI definition, all patients included in this study had a score of at least 1 due to their non-proliferative diabetic retinopathy status. As an additional severity factor, insulin use with prescription days covered counted as time on/off the drug within the model. Finally, the laboratory values that were assessed and updated within the model were haemoglobin 
A1c and estimated glomerular filtration rate (eGFR; calculated by the Modification of Diet in Renal Disease Study methodology; $\geq 90$ normal, 60-89 mild impairment, 45-59 moderate, 30-44 moderate/severe, 15-29 severe, $<15$ end stage disease) [12].

\section{Outcome measures}

A Cox proportional hazard model was created to determine the hazard of progressing to VTDR, which is a composite end point of either PDR or DME. Additional models were run to assess PDR and DME individually. Both PDR and DME were defined as a new diagnosis or treatment after the index date. To avoid confusion between DME and CME, or cystoid macular oedema, patients who had any form of intraocular surgery (e.g. vitrectomy, cataract surgery, trabeculectomy, etc.) were excluded from analysis for 120 days following surgery. Patients were allowed to return to the analysis after that time frame. To increase the likelihood that these were incident cases of VTDR, any patient who met VTDR definition within two years of entry into the insurance plan was excluded from analysis. Patients were followed until the end of eligibility or when they were censored. Censoring occurred if they had any diagnosis of a disease or treatment of exclusion noted earlier. Similarly, in considering the PDR and DME models separately, patients were censored from one once they met criteria for the other (i.e. censored in the PDR model if DME criteria were met). In addition, a sensitivity analysis was performed in which only patients with complete lab value data were assessed. $P$ values $<0.05$ were considered significant. Statistical analysis was performed using SAS (version 9.4; SAS Institute Inc., Cary, NC).

\section{Results}

After inclusion and exclusion criteria were applied, a total of 69,982 NPDR patients were analysed (Fig. 1). During the follow up period, 12,770 patients progressed to VTDR (18.25\%), 2162 to PDR (3.09\%), 10,322 to DME (14.75\%). The baseline characteristics of these patients are demonstrated in Table 1. Patients who progressed to VTDR were younger at baseline (No Progression 61.7 years vs. VTDR 60.5 years) and more likely to be female (No Progression $46.5 \%$ vs. VTDR $49.1 \%$ ) and White (No Progression $54.3 \%$ vs VTDR 56.9\%) or Black (No Progression $15.6 \%$ vs. VTDR $18.3 \%$ ). Of note, $p<0.001$ for all comparisons.

After controlling for all other covariates, both mild and $\mathrm{mod} / \mathrm{sev}$ anaemia were associated with an increased hazard ratio for progression to VTDR (mild $\mathrm{HR}=1.10$, 95\% CI:1.04-1.16, $\quad p<0.001 ; \quad \mathrm{mod} / \mathrm{sev} \quad \mathrm{HR}=1.20, \quad 95 \%$ CI:1.12-1.29, $p<0.001)$. Increased hazards were also found for anaemia and the individual components of VTDR: PDR (mild HR $=1.29,95 \%$ CI:1.13-1.46, $p<0.001 ; \mathrm{mod} / \mathrm{sev}$ $\mathrm{HR}=1.43,95 \% \mathrm{CI}: 1.21-1.69, p<0.001$ ) and DME (mild $\mathrm{HR}=1.06,95 \%$ CI:1.00-1.13, $p<0.001 ; \mathrm{mod} / \mathrm{sev} \mathrm{HR}=$ $1.14,95 \%$ CI:1.05-1.24, $p<0.001)$. ODCs were also shown to be associated with VTDR. These included both chronic pulmonary disease ( $\mathrm{HR}=1.10,95 \% \mathrm{CI}: 1.06-1.14, p<$ $0.001)$ and history of blood disorder/cancer $(\mathrm{HR}=1.28$, 95\% CI: $1.16-1.40, p<0.001)$. In both instances, however, this association appeared to be driven mostly by DME (chronic pulmonary disease-DME $\mathrm{HR}=1.15, \quad 95 \%$ CI:1.10-1.20, $\quad p<0.001 ; \quad$ blood disorder/cancer-DME $\mathrm{HR}=1.32$, 95\% CI:1.20-1.46, $p<0.001)$. As such, the individual associations between chronic pulmonary disease and blood disorder/cancer with PDR were not significant (chronic pulmonary disease-PDR $\mathrm{HR}=0.94, \quad 95 \%$ CI:0.85-1.04, $p=0.22$; blood disorder/cancer-DME HR = $1.04,95 \%$ CI:0.81-1.35, $p=0.74$ ). Table 2 contains the full results of the multivariate analyses.

The DCSI also showed a strong association between higher indices and increased hazards for VTDR (index score of $2-3 \mathrm{HR}=1.22$, 95\% CI:1.15-1.29; score of $4 \mathrm{HR}=1.40$, 95\% CI:1.31-1.50; score of $5+\quad \mathrm{HR}=1.62, \quad 95 \%$ CI:1.52-1.72, all $p<0.001$ for all comparisons), PDR (score of 2-3 HR: 1.26, 95\% CI:1.09-1.46, score of $4 \mathrm{HR}=1.41$, 95\% CI:1.18-1.67, score of $5+\mathrm{HR}=1.99, \quad 95 \%$ CI:1.71-2.33, $p<0.001$ for all comparisons), and DME (score of $2-3 \mathrm{HR}=1.20,95 \% \mathrm{CI}: 1.13-1.28$, score of 4 $\mathrm{HR}=1.38,95 \% \mathrm{CI}: 1.28-1.49$, score of $5+\mathrm{HR}=1.51,95 \%$ CI:1.41-1.62, $p<0.001$ for all comparisons). Other known risk factors also showed an increased hazard for progression to VTDR, PDR and DME, including increased haemoglobin A1c, hypertension, insulin use and worsening eGFR (HR > 1.00 and $p<0.001$ for all comparisons; Table 2).

\section{Discussion}

Anaemia, by definition, denotes a decreased haemoglobin concentration and is a marker of reduced oxygen carrying capacity. The current study supports the pathophysiological understanding of reduced oxygen carrying capacity aggravating tissue hypoxia, which is manifested as an association with progression to VTDR. Aside from anaemia, other diseases we deemed as oxygen-delivery-related comorbidities, or ODCs, such as chronic pulmonary disease and history of blood disorder/cancer, also showed increased hazards for progression to VTDR.

The pathophysiology of diabetic retinopathy is characterized by functional and structural abnormalities of the retinal microvasculature. Due to a lack of innervation by sympathetic nerve fibres within retinal vessels, diminished autoregulation of retinal blood flow in diabetes leads to 


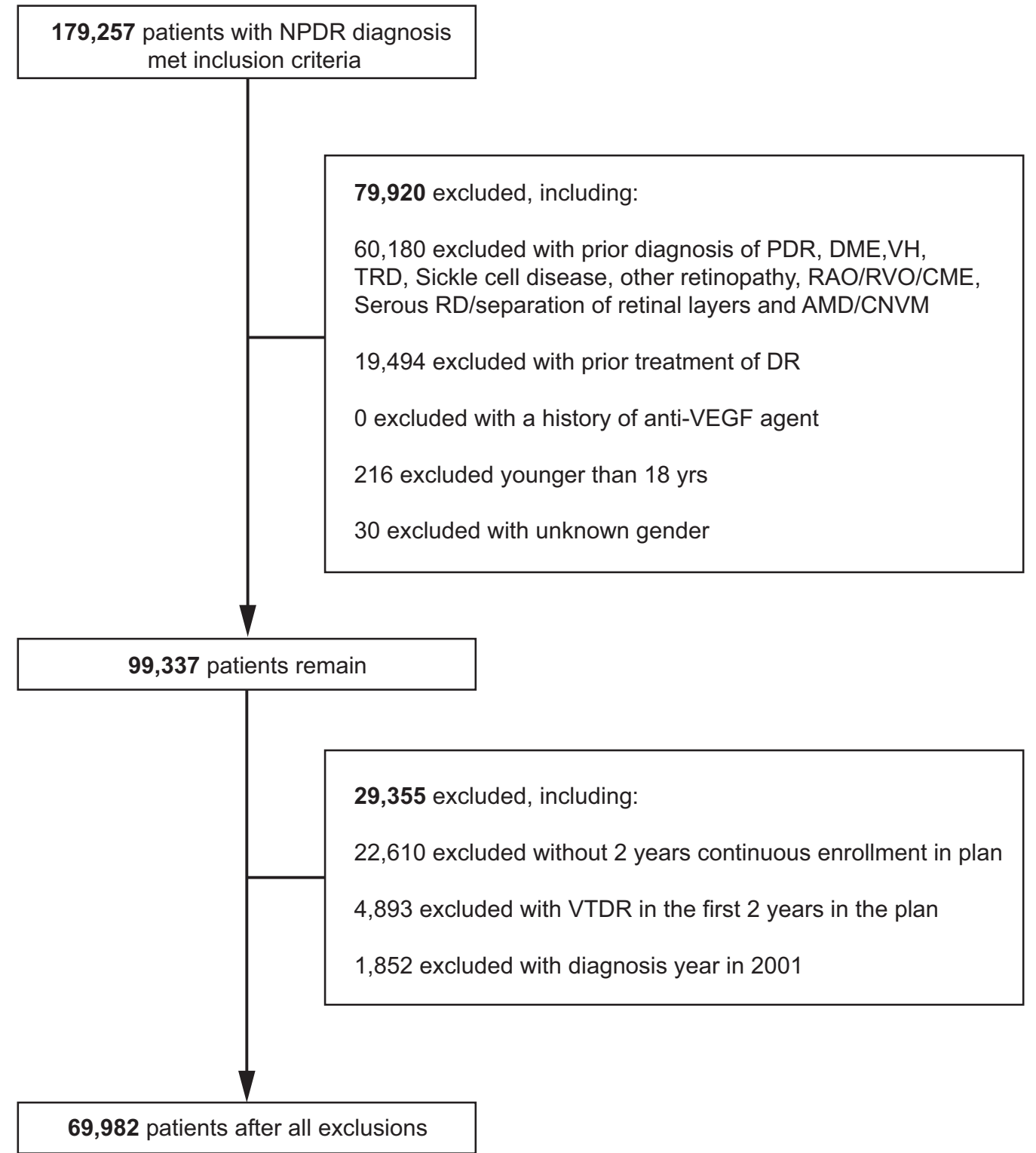

Fig. 1 Selection of patient subjects for analyses. NPDR nonproliferative diabetic retinopathy, PDR proliferative diabetic retinopathy, DME diabetic macular edema, VH vitreous hemorrhage, TRD tractional retinal detachment, RAO retinal arterial occlusion, RVO retinal venous occlusion, CME cystoid macular edema, RD retinal

vasodilation and increased permeability [13-15]. As both retinal capillary pericytes and endothelial cells undergo apoptosis under hyperglycaemic conditions, capillary nonperfusion develops, followed by breakdown of blood-retinal barrier [16]. Multiple hyperglycaemia-induced aberrant metabolic pathways lead to oxidative stress and formation of advanced glycation end-products, which further aggravate retinal hypoxia $[17,18]$. Acute hypoxia rapidly activates retinal vascular endothelial cells to release inflammatory cytokines [17]. These inflammatory mediators are able to recruit and promote the activation and adherence of leukocytes [18], which further contribute to the detachment, AMD age-related macular degeneration, CNVM choroidal neovascular membrane, DR diabetic retinopathy, VEGF vascular endothelial growth factor, VTDR vision-threatening diabetic retinopathy

obstruction of retinal capillaries. Chronic hypoxia then induces hypoxia inducible factor-1, a key transcription factor of vascular endothelial growth factor $[19,20]$. The expression of various angiogenic growth factors results in the characteristic retinal neovascularization associated with PDR [20]. Taken together, diabetic retinopathy progression is, in large part, driven by insufficient retinal tissue oxygenation, which is determined by a combination of capillary blood flow, haemoglobin concentration and haemoglobin oxygen affinity [21].

Despite a clear pathophysiologic link between oxygenation and diabetic retinopathy progression, few studies 
Table 1 Comparison of baseline characteristics between nonproliferative diabetic retinopathy patients who did not progress and those who did progress to vision threatening diabetic retinopathy (VTDR)

\begin{tabular}{|c|c|c|}
\hline & $\begin{array}{l}\text { No progression } \\
\text { to VTDR }(N= \\
57,212)\end{array}$ & $\begin{array}{l}\text { Progressed to } \\
\text { VTDR }(N= \\
12,770)\end{array}$ \\
\hline Age (Mean (SD)) & $61.7(12.9)$ & $60.5(12.1)$ \\
\hline \multicolumn{3}{|l|}{ Gender } \\
\hline Female & $26,617(46.5 \%)$ & $6,271(49.1 \%)$ \\
\hline Male & $30,595(53.5 \%)$ & $6499(50.9 \%)$ \\
\hline \multicolumn{3}{|l|}{ Race } \\
\hline White & $31,066(54.3 \%)$ & $7266(56.9 \%)$ \\
\hline Black & $8944(15.6 \%)$ & $2334(18.3 \%)$ \\
\hline Asian & $2835(5.0 \%)$ & $445(3.5 \%)$ \\
\hline Hispanic & $9004(15.7 \%)$ & $1837(14.4 \%)$ \\
\hline Unknown & $5363(9.4 \%)$ & $888(7.0 \%)$ \\
\hline Hypertension & $47,999(83.9 \%)$ & $10,952(85.8 \%)$ \\
\hline Hypercholesterolemia & $48,739(85.2 \%)$ & $11,003(86.2 \%)$ \\
\hline Previous ischemic stroke/TIA & $4729(8.3 \%)$ & $1111(8.7 \%)$ \\
\hline $\begin{array}{l}\text { Previous intracerebral } \\
\text { hemorrhage }\end{array}$ & $323(0.6 \%)$ & $39(0.3 \%)$ \\
\hline Chronic liver disease & $464(0.8 \%)$ & $95(0.7 \%)$ \\
\hline Peripheral vascular disease & $10,734(18.8 \%)$ & $2523(19.8 \%)$ \\
\hline Any malignancy & $5868(10.3 \%)$ & $1315(10.3 \%)$ \\
\hline \multicolumn{3}{|l|}{ eGFR (kidney function) } \\
\hline Normal $(\geq 90)$ & $11127(19.4 \%)$ & $2254(17.7 \%)$ \\
\hline Mild (60-89) & $22850(39.9 \%)$ & $4861(38.1 \%)$ \\
\hline Moderate (45-59) & $7471(13.1 \%)$ & $1648(12.9 \%)$ \\
\hline Mod/severe (30-44) & $3771(6.6 \%)$ & $912(7.1 \%)$ \\
\hline Severe (15-29) & $1270(2.2 \%)$ & $334(2.6 \%)$ \\
\hline $\begin{array}{l}\text { End stage disease or } \\
\text { dialysis }(<15)\end{array}$ & $4803(8.4 \%)$ & $1478(11.6 \%)$ \\
\hline Unknown & $5920(10.3 \%)$ & $1283(10.0 \%)$ \\
\hline $\begin{array}{l}\text { Hemoglobin A1c } \\
\text { (Mean (SD)) }\end{array}$ & $7.1(2.9)$ & $7.1(3.3)$ \\
\hline Insulin Use & $11529(20.2 \%)$ & $2824(22.1 \%)$ \\
\hline \multicolumn{3}{|l|}{ Diabetes Comp Sev Index } \\
\hline 1 & $17736(31.0 \%)$ & $3567(27.9 \%)$ \\
\hline $2-3$ & $18168(31.8 \%)$ & $4111(32.2 \%)$ \\
\hline 4 & $6330(11.1 \%)$ & $1586(12.4 \%)$ \\
\hline $5-12$ & $14978(26.2 \%)$ & $3506(27.5 \%)$ \\
\hline \multicolumn{3}{|l|}{ Anaemia } \\
\hline $\begin{array}{l}\text { Normal }(\mathrm{M} \geq 13.0 \text {, } \\
\mathrm{W} \geq 12.0)\end{array}$ & $24,090(42.1 \%)$ & $4916(38.5 \%)$ \\
\hline $\begin{array}{l}\text { Mild (M:11.0-12.9, } \\
\text { W:11.0-11.9) }\end{array}$ & $5829(10.2 \%)$ & $1362(10.7 \%)$ \\
\hline Mod/severe $(\leq 10.9)$ & $2684(4.7 \%)$ & $637(5.0 \%)$ \\
\hline Unknown & $24,609(43.0 \%)$ & $5855(45.8 \%)$ \\
\hline Chronic pulmonary disease & $12,656(22.1 \%)$ & $3003(23.5 \%)$ \\
\hline $\begin{array}{l}\text { History of blood disorder/ } \\
\text { cancer }\end{array}$ & $1160(2.0 \%)$ & $290(2.3 \%)$ \\
\hline
\end{tabular}

have examined the risk posed by anaemia. One potential reason for this could be the strong association between anaemia and chronic kidney disease (CKD) within diabetics, which could have caused researchers to argue that controlling for kidney disease likely also controls for kidney disease-related anaemia. However, the results of this study, which accounted for kidney function by using timedependent values for eGFR, strongly refute this idea. Furthermore, pathogenic factors independent of CKD may also cause anaemia, including inflammation, nutritional deficiency and concomitant autoimmune diseases [22, 23]. To assume diabetic kidney disease is the only cause of anaemia ignores these other considerations.

In addition to corroborating the idea that anaemia's effect on progression to VTDR is independent of kidney disease, our study is also consistent with findings from the only other published study examining the risk of progression to DME in anaemic patients [6]. Lastly, to the best of the authors' knowledge, this is the first study to assess the impact of other oxygen delivery-related diseases, history of chronic pulmonary disease, and blood disorder/cancers.

This study offers two distinct advantages over previous studies in this area. The first and most obvious one is the size of the cohort studied. We were able to evaluate 69,982 NPDR patients for their progression to VTDR, including 2162 that progressed to PDR and 10,322 to DME. This is the largest study in terms of total population and outcomes for each of these disease states. The other advantage of this study is the time-dependent manner of this study, which had not been previously utilized in this area and allowed for changes in patient characterization each and every time new information was found. Updating variables in this manner offers better control for values that can naturally fluctuate with time and can offer truer associations for these variables. This is particularly true compared to studies that measure a variable once at baseline and then observe patients for an outcome over long periods of time [24]. Furthermore, in contrast to the two previous cross-sectional studies [5, 6], our cohort design allowed for a temporal relationship to be established, demonstrating that the anaemia was present prior to diagnosis. While this temporal relationship does not prove causality alone, it is a necessary requirement for the creation of any causal pathway linking anaemia to VTDR.

The results of this study must also be considered concurrent with its potential limitations. One of the primary limitations of this study is the lack of ability to control for duration of disease, one of the strongest known predictors for progression of DR [1, 2, 5]. However, it is well known that systemic insults from diabetes accrue with time. For this reason, we incorporated the DCSI into our model. This is an administrative claims-based measure that accounts for the systemic complications that frequently occur within 
Table 2 Full model results showing hazard ratios (HR) associated with progression from NPDR to VTDR, PDR, or DME using a time-dependent multivariate Cox regression model

\begin{tabular}{|c|c|c|c|c|c|c|}
\hline \multirow[b]{2}{*}{ Variable } & \multicolumn{2}{|c|}{$\begin{array}{l}\text { Progression to vision- } \\
\text { threatening diabetic } \\
\text { retinopathy (VTDR) }\end{array}$} & \multicolumn{2}{|c|}{$\begin{array}{l}\text { Progression to proliferative } \\
\text { diabetic retinopathy (PDR) }\end{array}$} & \multicolumn{2}{|c|}{$\begin{array}{l}\text { Progression to diabetic } \\
\text { macular edema (DME) }\end{array}$} \\
\hline & $\mathrm{HR}(95 \% \mathrm{CI})$ & $P$-value & HR $(95 \% \mathrm{CI})$ & $P$-value & $\mathrm{HR}(95 \% \mathrm{CI})$ & $P$-value \\
\hline Anaemia & & $<0.001$ & & $<0.001$ & & $<0.001$ \\
\hline Normal $(\mathrm{M} \geq 13.0, \mathrm{~W} \geq 12.0)$ & Reference & & Reference & & Reference & \\
\hline Mild (M:11.0-12.9, W:11.0-11.9) & $1.10(1.04,1.16)$ & & $1.29(1.13,1.46)$ & & $1.06(1.00,1.13)$ & \\
\hline Mod/Severe $(\leq 10.9)$ & $1.20(1.12,1.29)$ & & $1.43(1.21,1.69)$ & & $1.14(1.05,1.24)$ & \\
\hline Unknown & $0.99(0.94,1.03)$ & & $1.05(0.94,1.18)$ & & $0.97(0.92,1.02)$ & \\
\hline History of blood disorder/malignancy & $1.28(1.16,1.40)$ & $<0.001$ & $1.04(0.81,1.35)$ & 0.74 & $1.32(1.20,1.46)$ & $<0.001$ \\
\hline Chronic pulmonary disease & $1.10(1.06,1.14)$ & $<0.001$ & $0.94(0.85,1.04)$ & 0.22 & $1.15(1.10,1.20)$ & $<0.001$ \\
\hline Any Malignancy & $1.16(1.10,1.22)$ & $<0.001$ & $0.89(0.78,1.03)$ & 0.12 & $1.23(1.16,1.30)$ & $<0.001$ \\
\hline Hypercholesterolemia & $1.16(1.08,1.25)$ & $<0.001$ & $0.93(0.79,1.10)$ & 0.40 & $1.25(1.15,1.36)$ & $<0.001$ \\
\hline Hypertension & $1.25(1.16,1.34)$ & $<0.001$ & $1.31(1.11,1.56)$ & 0.002 & $1.23(1.14,1.34)$ & $<0.001$ \\
\hline Hemoglobin A1c & $1.04(1.03,1.05)$ & $<0.001$ & $1.09(1.07,1.11)$ & $<0.001$ & $1.02(1.01,1.03)$ & 0.002 \\
\hline Insulin Use & $1.21(1.16,1.26)$ & $<0.001$ & $1.63(1.48,1.79)$ & $<0.001$ & $1.21(1.15,1.26)$ & $<0.001$ \\
\hline eGFR & & $<0.001$ & & $<0.001$ & & $<0.001$ \\
\hline Normal $(\geq 90)$ & Reference & & Reference & & Reference & \\
\hline Mild (60-89) & $1.03(0.98,1.08)$ & & $1.20(1.05,1.36)$ & & $0.98(0.93,1.04)$ & \\
\hline Moderate (45-59) & $1.03(0.96,1.10)$ & & $1.36(1.15,1.60)$ & & $0.94(0.87,1.01)$ & \\
\hline Mod/severe (30-44) & $1.06(0.98,1.15)$ & & $1.76(1.46,2.12)$ & & $0.91(0.83,0.99)$ & \\
\hline Severe $(15-29)$ & $1.14(1.02,1.27)$ & & $2.00(1.58,2.53)$ & & $0.96(0.84,1.08)$ & \\
\hline End stage disease or dialysis $(<15)$ & $1.37(1.25,1.50)$ & & $2.20(1.79,2.71)$ & & $1.12(1.01,1.24)$ & \\
\hline Unknown & $1.12(1.02,1.23)$ & & $1.41(1.13,1.76)$ & & $1.04(0.94,1.15)$ & \\
\hline Diabetic Complications Severity Index (DCSI) & & $<0.001$ & & $<0.001$ & & $<0.001$ \\
\hline 1 & Reference & & Reference & & Reference & \\
\hline$[2,3]$ & $1.22(1.15,1.29)$ & & $1.26(1.09,1.46)$ & & $1.20(1.13,1.28)$ & \\
\hline 4 & $1.40(1.31,1.50)$ & & $1.41(1.18,1.67)$ & & $1.38(1.28,1.49)$ & \\
\hline$[5,12]$ & $1.62(1.52,1.72)$ & & $1.99(1.71,2.33)$ & & $1.51(1.41,1.62)$ & \\
\hline
\end{tabular}

diabetics and has been found to be a better predictor of clinically meaningful outcomes for diabetes than duration of disease [11]. While we cannot prove this measure sufficiently accounts for duration with respect to retinopathy progression, given its proven validity in other diabetic outcomes and the increasing risks for VTDR, PDR and DME that correlated with increasing DCSI score within our results, we believe this measure to be a valuable tool for predicting DR progression. The other limitation that needs to be considered is the inability to verify any individual diagnosis due to the de-identified nature of the database. While unable to fully eliminate this issue, it also should be noted that previous work has studied the validity of diagnosis and treatment codes for diabetic retinopathy and have found them to be highly representative of the clinical chart [7-9]. Similarly, the current study was performed in a single large insurance database. Given the most common reason to be included in this database is obtaining insurance through a
US employer, it is unclear if these results would be generalizable to other populations that are uninsured, covered by other insurance companies, or outside of the healthcare system altogether. Next, due to the majority of data being accrued in ICD9 diagnosis code form, we are unable to distinguish between right, left and/or bilateral progression of disease. Lastly, several thousand people were excluded for not having enough time in the database, limiting the ability to capture complete data on them for inclusion. Due to their incomplete data, it is unclear how the inclusion of these patients would have affected the final results.

This study offers new evidence that anaemia and other oxygen-delivery-related comorbidities are risk factors for DR progression to its vision-threatening forms. Future investigations of risk factors for disease progression or predicting which NPDR patients will progress to VTDR should account for these variables. Importantly, however, the small effect size seen within some of the comparisons 
suggests that further work is warranted before these variables become clinical targets for reduction of disease progression.

\section{Summary}

\section{What was known before}

- Although the association between anaemia and diabetic retinopathy has been studied, anaemia is a less frequently recognized risk factor of non-proliferative diabetic retinopathy (NPDR) progression.

- The idea that controlling for kidney disease likely controls for anaemia-related complications in diabetics is seemingly reasonable but inaccurate.

\section{What this study adds}

- Large scale analyses of NPDR progression risk factors are performed in a time-varying manner, with updates each time new information was available for the variable in question.

- Anaemia as a risk factor of diabetic retinopathy progression is independent of chronic kidney disease in diabetic patients.

Funding This research is funded by National Institutes of Health K23 Award (1K23EY025729-01) and University of Pennsylvania Core Grant for Vision Research (2P30EY001583). The content is solely the responsibility of the authors and does not necessarily represent the official views of the NIH. Additional funding was provided by Research to Prevent Blindness and the Paul and Evanina Mackall Foundation. Funding from each of the above sources was received in the form of block research grants to the Scheie Eye Institute. None of the organizations had any role in the design or conduction of the study.

\section{Compliance with ethical standards}

Conflict of interest The authors declare that they have no conflict of interest.

Publisher's note Springer Nature remains neutral with regard to jurisdictional claims in published maps and institutional affiliations.

\section{References}

1. Thomas M, Tsalamandris C, MacIsaac R, Jerums G. Anaemia in diabetes: an emerging complication of microvascular disease. Curr Diabetes Rev. 2005;1:107-26.

2. Rand LI, Prud'homme GJ, Ederer F, Canner PL. Factors influencing the development of visual loss in advanced diabetic retinopathy. Diabetic Retinopathy Study (DRS) Report No. 10. Investig Ophthalmol Vis Sci. 1985;26:983-91.
3. Qiao Q, Keinänen-Kiukaanniemi S, Läärä E. The relationship between hemoglobin levels and diabetic retinopathy. J Clin Epidemiol. 1997;50:153-8. https://doi.org/10.1016/S0895-4356(96) 00335-6.

4. Davis MD, Fisher MR, Gangnon RE, Barton F, Aiello LM, Chew $\mathrm{EY}$, et al. Risk factors for high-risk proliferative diabetic retinopathy and severe visual loss: Early Treatment Diabetic Retinopathy Study Report \#18. Investig Ophthalmol Vis Sci. 1998;39:233-52.

5. Irace C, Scarinci F, Scorcia V, Bruzzichessi D, Fiorentino R, Randazzo G, et al. Association among low whole blood viscosity, haematocrit, haemoglobin and diabetic retinopathy in subjects with type 2 diabetes. Br J Ophthalmol. 2011;95:94. https://doi.org/ 10.1136/bjo.2009.172601.

6. Traveset A, Rubinat E, Ortega E, Alcubierre N, Vazquez B, Hernández $\mathrm{M}$, et al. Lower hemoglobin concentration is associated with retinal ischemia and the severity of diabetic retinopathy in type 2 diabetes. J Diabetes Res. 2016;2016:3674946. https://doi.org/10.1155/2016/3674946.

7. Muir KW, Gupta C, Gill P, Stein JD. Accuracy of international classification of diseases, ninth revision, clinical modification billing codes for common ophthalmic conditions. JAMA Ophthalmol. 2013;131:119-20. https://doi.org/10.1001/jamaophtha lmol.2013.577.

8. Bearelly S, Mruthyunjaya P, Tzeng JP, Suñer IJ, Shea AM, Lee $\mathrm{JT}$, et al. Identification of patients with diabetic macular edema from claims data: a validation study. Arch Ophthalmol. 2008;126:986-9. https://doi.org/10.1001/archopht.126.7.986.

9. Lau M, Prenner JL, Brucker AJ, VanderBeek BL. Accuracy of billing codes used in the therapeutic care of diabetic retinopathy. JAMA Ophthalmol. 2017;135:791-4. https://doi.org/10.1001/ja maophthalmol.2017.1595.

10. WHO. Haemoglobin concentrations for the diagnosis of anaemia and assessment of severity. Vitamin and Mineral Nutrition Information System. World Health Organization: Geneva; 2011 (WHO/NMH/NHD/MNM/11.1). http://www.who.int/vmnis/ indicators/haemoglobin.pdf. Accessed 30 Mar 2019.

11. Young BA, Lin E, Von Korff M, Simon G, Ciechanowski P, Ludman EJ, et al. Diabetes complications severity index and risk of mortality, hospitalization, and healthcare utilization. Am J Manag Care. 2008;14:15-23.

12. Levey AS, Bosch JP, Lewis JB, Greene T, Rogers N, Roth D, et al. A more accurate method to estimate glomerular filtration rate from serum creatinine: a new prediction equation. Ann Intern Med. 1999;130:461-70. https://doi.org/10.7326/0003-4819-1306-199903160-00002.

13. Patz A. Clinical and experimental studies on retinal neovascularization: XXXIX Edward Jackson Memorial Lecture. Am J Ophthalmol. 1982;94:715-43. https://doi.org/10.1016/0002-9394 (82)90297-5.

14. Sinclair SH. Macular retinal capillary hemodynamics in diabetic patients. Ophthalmology. 1991;98:1580-6. https://doi.org/10. 1016/S0161-6420(91)32084-0.

15. McDougal DH, Gamlin PD. Autonomic control of the eye. Compr Physiol. 2015;5:439-73. https://doi.org/10.1002/cphy.c140014.

16. Li W, Yanoff M, Liu X, Ye X. Retinal capillary pericyte apoptosis in early human diabetic retinopathy. Chin Med J. 1997; 110:659-63.

17. Lu M, Kuroki M, Amano S, Tolentino M, Keough K, Kim I, et al. Advanced glycation end products increase retinal vascular endothelial growth factor expression. $\mathrm{J}$ Clin Investig. 1998;101:1219-24. https://doi.org/10.1172/JCI1277.

18. Michiels C, Arnould T, Remacle J. Endothelial cell responses to hypoxia: initiation of a cascade of cellular interactions. Biochim Biophys Acta. 2000;1497:1-10. 
19. Kurihara T, Westenskow PD, Friedlander M. Hypoxia-inducible factor (HIF)/vascular endothelial growth factor (VEGF) signaling in the retina. Adv Exp Med Biol. 2014;801:275-81. https://doi. org/10.1007/978-1-4614-3209-8_35.

20. Yu Y, Zhang J, Zhu R, Zhao R, Chen J, Jin J, et al. The profile of angiogenic factors in vitreous humor of the patients with proliferative diabetic retinopathy. Curr Mol Med. 2017;17:280-6. https://doi.org/10.2174/1566524017666171106111440.

21. Ditzel J. Affinity hypoxia as a pathogenetic factor of microangiopathy with particular reference to diabetic retinopathy. Acta Endocrinol Suppl. 1980;238:39-55.
22. Craig KJ, Williams JD, Riley SG, Smith H, Owens DR, Worthing $\mathrm{D}$, et al. Anaemia and diabetes in the absence of nephropathy. Diabetes Care. 2005;28:1118-23.

23. Friedman EA, Brown CD, Berman DH. Erythropoietin in diabetic macular edema and renal insufficiency. Am J Kidney Dis. 1995;26:202-8. https://doi.org/10.1016/0272-6386 (95)90175-2.

24. Fisher LD, Lin DY. Time-dependent covariates in the cox proportional-hazards regression model. Annu Rev Public Health. 1999;20:145-57. https://doi.org/10.1146/annurev.publhea 1th.20.1.145. 